\title{
Does neoadjuvant chemotherapy increase breast conservation in operable breast cancer: an Egyptian experience
}

\author{
N Abdel-Bary ${ }^{1}$, AF EI-Kased ${ }^{2}$ and HAZ Aiad $^{3}$ \\ ${ }^{1}$ Department of Clinical Oncology, Faculty of Medicine, Menofia University, Egypt \\ ${ }^{2}$ Department of Surgery, Faculty of Medicine, Menofia University, Egypt \\ ${ }^{3}$ Department of Pathology, Faculty of Medicine, Menofia University, Egypt
}

\begin{abstract}
Introduction: The role of adjuvant chemotherapy in breast cancer is well established, as are its indications. Likewise, the role of neoadjuvant chemotherapy in locally advanced breast cancer is well established. The use of neoadjuvant chemotherapy in operable breast cancer has only recently become of interest to researchers.

Patients and methods: This study included 34 cases of operable breast cancer that were given four cycles of neoadjuvant chemotherapy in the form of FEC100 then subjected to surgery. The surgery done was either breast conserving surgery or modified radical mastectomy. All patients completed the treatment regimen and no patients were excluded from the study. All surgical specimens were studied pathologically for chemotherapy effect.
\end{abstract}

Results: An overall objective response was observed in $70.6 \%$ of the patients. Seven patients (20.6\%) experienced a clinical complete response (cCR), 17 patients $(50.0 \%)$ had partial response, nine patients $(26.5 \%)$ had no change of their disease and only one patient had disease progression. Of the seven patients who had a CCR, only four patients (11.8\%) had pathologic complete response (pCR), while pCR for the whole group was 14.7\%(5/34). Tumour size of more than $2 \mathrm{~cm}$ was observed in 28 patients (82.4\%) at time of presentation, while tumour size of $2 \mathrm{~cm}$ or less was seen in six patients (17.6\%) only. After completion of the course of chemotherapy, 23 patients (67.6\%) were observed to have tumours of $2 \mathrm{~cm}$ or less that allowed for less extensive resections. Twenty-three patients underwent breast conservative surgery (67.6\%) while modified radical mastectomy was performed in 11 patients (32.4\%).

Conclusion: The use of neoadjuvant chemotherapy in operable breast cancer in this study was associated with tumour and axillary downstaging, which increased the proportion of cases undergoing breast conservation, with acceptable side effects and reasonable cost. During the limited follow-up time of this study no loco regional recurrences were recorded and one distant treatment failure was recorded. Its impact if any on overall or disease-free survival was not addressed in this study. Larger multi-centre randomized studies with a long follow-up are needed to compare the overall and disease-free survival benefit of this treatment modality, especially in different subtypes stratified by pathological response.

Published: 09/04/2009

Received: 07/11/2008

ecancer 2009, 3:104 DOI: 10.3332/ecancer.2008.104

Copyright: (c) the authors; licensee ecancermedicalscience. This is an Open Access article distributed under the terms of the Creative Commons Attribution License (http://creativecommons.org/licenses/by/2.0), which permits unrestricted use, distribution, and reproduction in any medium, provided the original work is properly cited.

Competing Interests: The authors have declared that no competing interests exist.

Correspondence to N Abdel-Bary. Email: nbary11@yahoo.com 


\section{Introduction}

Trials that studied the role of adjuvant chemotherapy in the management of primary operable breast cancer conducted during the 1970s and 1980s showed significant improvements in progression-free and overall survival [1]. Conventionally, adjuvant systemic therapy is administered after local treatment in early breast cancer [2]. The role of neoadjuvant chemotherapy and endocrine therapy in locally advanced breast cancer has also become well established [3]. However, since the introduction of conservative treatment modalities, there has been considerable interest in the efficacy of preoperative chemotherapy to decrease tumour size. One of the potential benefits of preoperative chemotherapy is the more frequent usage of breast-conserving treatment modalities [4]. Moreover, it has been hypothesized that preoperative chemotherapy may have a more powerful effect on survival compared with postoperative chemotherapy. It was found that animal models treated with chemotherapy or tamoxifen prior to surgical resection have improved survival, presumed as a result of a reduction in dissemination of cancer cells following surgery [57]. Successful early treatment with systemic therapy is consistent with the Goldie-Coldman hypothesis, whereby metastases are being treated prior to the emergence of chemoresistant mutant clones [8].

Although the merits of preoperative chemotherapy in the treatment of locally advanced breast cancer are well established, the feasibility of preoperative chemotherapy in early breast cancer is still a matter of discussion.

The European Organization for Research and Treatment of Cancer (EORTC) Breast Cancer Cooperative Group started a randomized trial in 1991 (EORTC trial 10902) to investigate the value of preoperative chemotherapy in early breast cancer and concluded that there is no difference in terms of overall survival or relapse-free survival between pre- and post-operative chemotherapy in early breast cancer. The data also concluded that, in such setting, preoperative chemotherapy allows better breast conservation. Survival data from the much larger NSABP B18 trial, with 1523 patients randomized to four cycles of adriamycin plus cyclophosphamide before or after surgery, have recently been reported with no significant differences in overall survival or relapse-free survival.

The study presented here assesses the clinical as well as pathological response of breast cancer to a preoperative FEC100 regimen (fluorouracil $500 \mathrm{mg} / \mathrm{m}^{2}$, epirubicin $100 \mathrm{mg} / \mathrm{m}^{2}$, cyclophosphamide $500 \mathrm{mg} / \mathrm{m}^{2}$ administered intravenously), and its significance in reducing the need for mastectomy and increasing the potential for more conservative breast surgery at the Menofia Oncology Hospital, Egypt.

\section{Patients and methods}

\section{Patient characteristics}

Thirty-four women with breast cancer attending the Department of Surgical Oncology at the Menofia Oncology Hospital were enrolled into this study from June 2004 to November 2006. Patients had primary operable breast cancer (T1 to T3, NO to 1 and $\mathrm{MO}$ ). Breast cancer was diagnosed by core-needle biopsy in all cases even if the patient had been previously diagnosed by fine-needle aspiration. A complete history was taken for all patients at the first presentation and a complete physical examination (including careful assessment of breast as well as axillary lymph nodes), routine laboratory tests, including CBC, liver and renal function tests, alkaline phosphatase, radiological tests, including a chest x-ray, pelvic-abdominal ultrasound, bilateral mammography with confirmatory breast ultrasound and complete echocardiography with assessment of the ejection fraction were carried out at this point and after the completion of the course of chemotherapy to assess treatment response. $\mathrm{CBC}$ was performed prior to each cycle of chemotherapy.

Exclusion criteria consisted of the following:

- more than 70 years old;

- lumpectomy or axillary nodal biopsy;

- bilateral breast cancer;

- previous treatment for breast cancer;

- $\quad$ presence of distant metastases;

- pregnancy or lactation at the time of diagnosis;

- previous or current other malignancies;

- World Health Organization performance status more than 2;

- active cardiac disease (including EF below 55\%);

- severe haematological, renal or hepatic abnormalities.

All patients gave informed consent before entering the trial. 


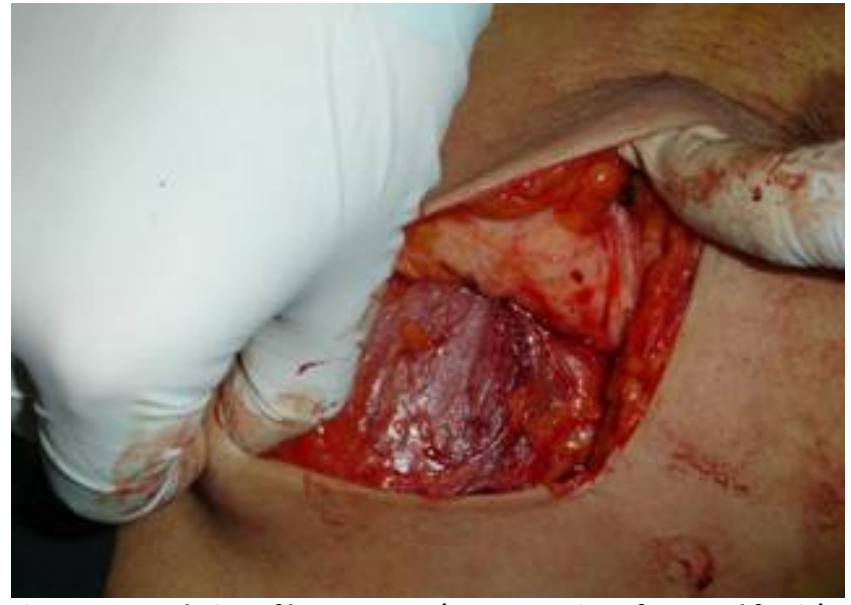

Figure 1: Completion of lumpectomy (note resection of pectoral fascia)

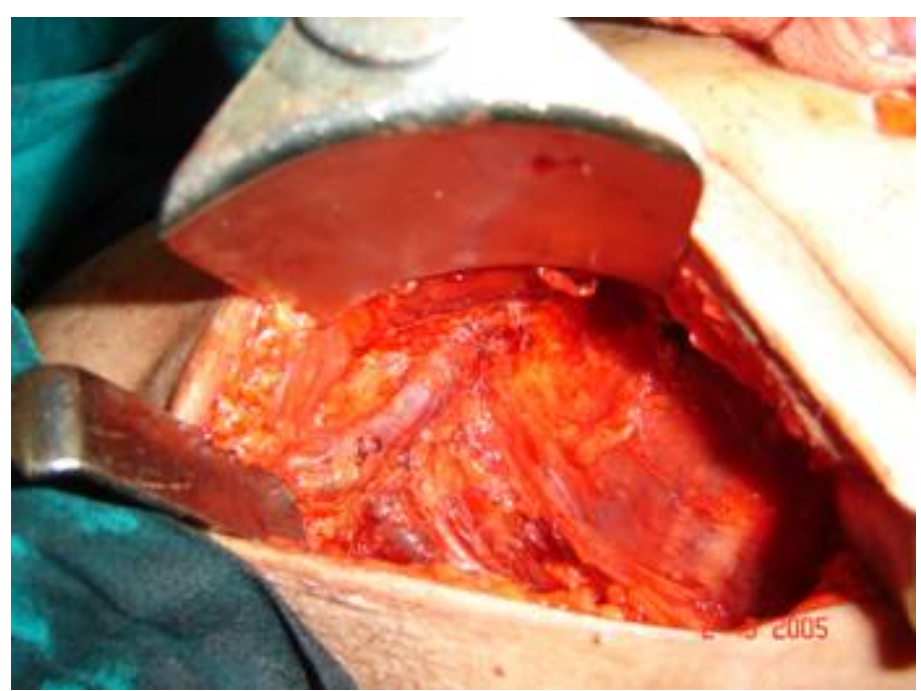

Figure 3: Full axillary dissection, showing vein and nerve to serratus anterior

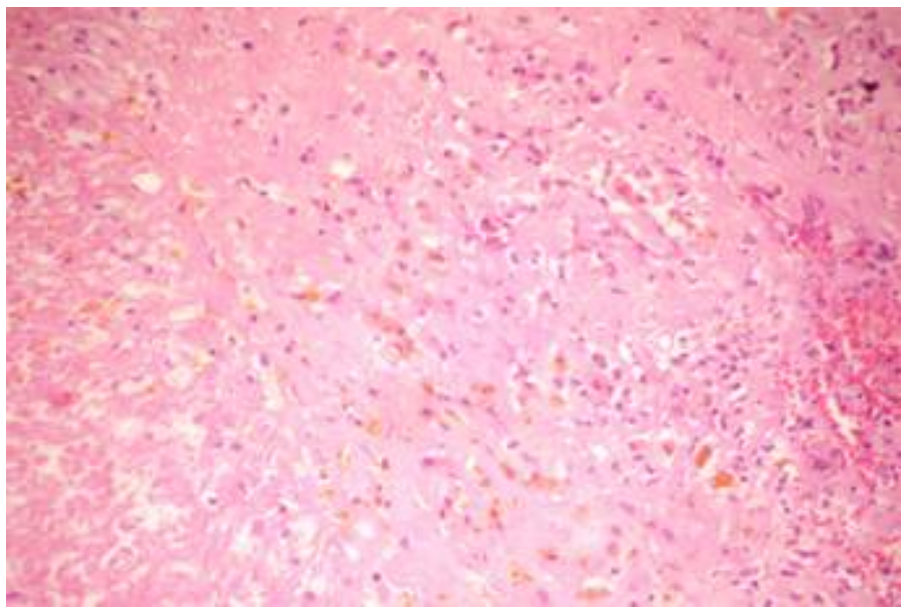

Figure 4b: High-power view of Figure 4a, showing from right to left fibrosis, aggregates of foamy histiocytes and necrosis (H\&E X400)

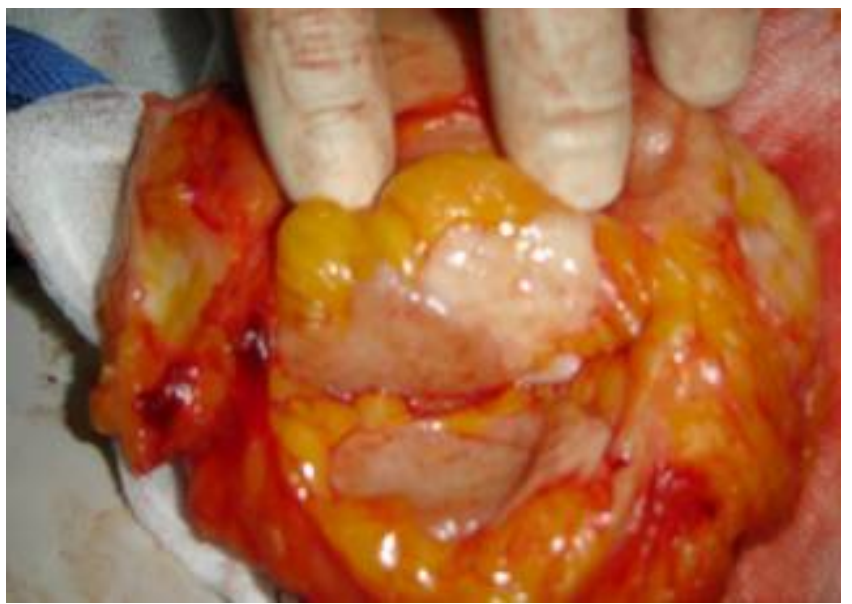

Figure 2: Bisected lumpectomy specimen with grossly adequate margins

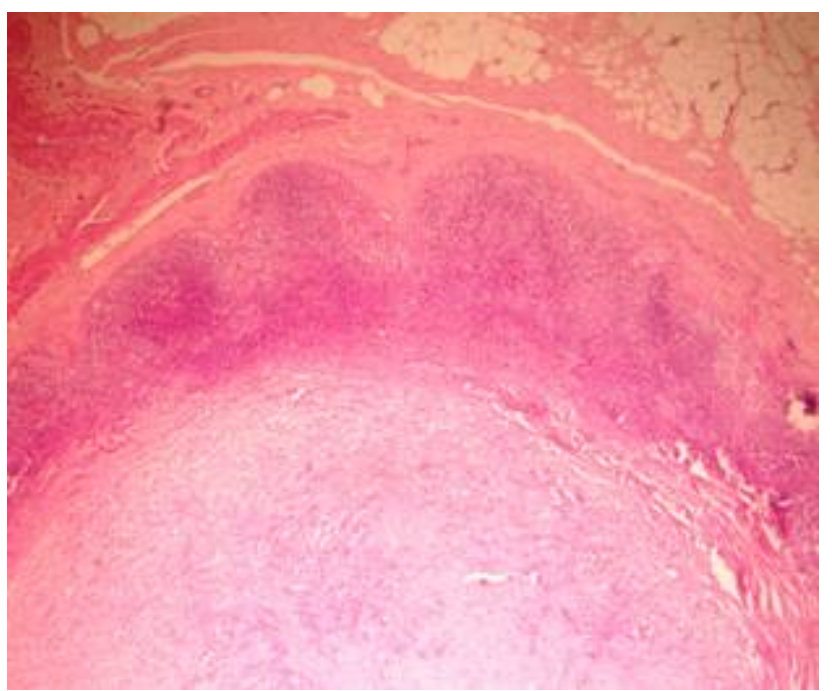

Figure 4a: Lymph node, showing partial replacement by evidence of tumour regression (H\&E X200) 


\section{Treatment}

Treatment consisted of four cycles of the FEC-100 regimen followed by surgery. Decision on the type of surgery (whether breast conserving surgery or mastectomy) was taken on the final assessment of the disease after the fourth cycle.

The chemotherapy regimen consisted of four cycles of preoperative fluorouracil $500 \mathrm{mg} / \mathrm{m}^{2}$, epirubicin $100 \mathrm{mg} / \mathrm{m}^{2}$ and cyclophosphamide $500 \mathrm{mg} / \mathrm{m}^{2}$ (FEC-100) administered intravenously, at three-week interval. Administration of FEC was delayed for a maximum of two weeks in the case of haematological, hepatic, renal or gastrointestinal toxicity. Dose modifications followed the guidelines stipulated by the EORTC Breast Cancer Cooperative Group [10].

Surgery was planned to be performed within 4-6 weeks of the fourth course of chemotherapy.

\section{Tumour response}

Clinical tumour size and nodal status were estimated before the start of chemotherapy as well as at the time of surgery by both palpation and mammography. The product of the two greatest perpendicular diameters was used to compare tumour size before and after chemotherapy, as defined by the International Union Against Cancer criteria [11]. A complete clinical response (CCR) was considered a complete disappearance of all clinically detectable malignant disease by palpation as well as mammography. The clinical response to chemotherapy was assessed before each cycle and at the time of surgery. If the tumour had become undetectable before completion of the four cycles of preoperative chemotherapy, chemotherapy was continued as outlined in the protocol. Clinical partial response was defined as $50 \%$ decrease in total tumour size after four cycles of chemotherapy at the time of surgery. An increase of $25 \%$ in tumour size after a minimum of two courses of preoperative chemotherapy was considered to be progressive disease (PD). In patients with clinically negative nodes at presentation, the development of palpable nodes during the administration of chemotherapy was considered evidence of PD. After a diagnosis of PD, patients immediately underwent surgery before completing the preoperative chemotherapy schedule. If patients did not meet one of the above-mentioned criteria after four cycles of chemotherapy, they were classified as having stable disease. Tumour specimens were examined pathologically to assess the response and compare clinical to pathologic response. If no signs of residual malignant cells at the primary site and axillary lymph nodes were seen with histological examination, this was scored as a pathologic complete response (pCR).

\section{Pathology}

The surgical specimens were resected and oriented according to a defined surgical protocol described in the UK Guidelines [12]. For the therapeutic wide local excisions, once in the laboratory, the entire surface of the specimen was stained so that the margins of excision could be easily determined. The system followed to record the clinical response to neoadjuvant therapy was that of the International Union Against Cancer [11]. The histopathological response to neoadjuvant therapy was assessed according to criteria suggested by Smith et al [13]. These authors suggest that the features of the primary tumour should be scored from $\mathrm{G} 1$ to $\mathrm{G} 5$ :

1. no reduction in overall numbers of tumour cells compared with pre-treatment core biopsy;

2. mild loss of tumour cells, but overall cellularity remaining high;

3. up to $90 \%$ reduction in tumour cells;

4. marked disappearance with only small clusters remaining;

5. no invasive tumour, in situ carcinoma or stromal reaction remaining.

Similarly, the lymph nodes are categorized as follows:
A true negative, no metastasis and no alterations;
B metastasis with no histological alterations;
C metastasis with alterations;
D no metastasis with alterations.

For classification into these categories, it was essential that an invasive tumour was identified. This necessitated the detection of abnormal fibroelastic breast stroma that was devoid of normal lobular units and contained foamy macrophages, a moderate numbers of fibroblasts and other mononuclear inflammatory cells. Grade 5 response was deemed to represent a pCR of the primary cancer.

Oestrogen and progesterone receptor status were ascertained in all specimens by immunohistochemical staining. In brief 5 $\mu \mathrm{m}$-thick sections of paraffin-embedded blocks were deparaffinized, dehydrated and then placed in citrate buffered 
Table 1: Patient characteristics at presentation

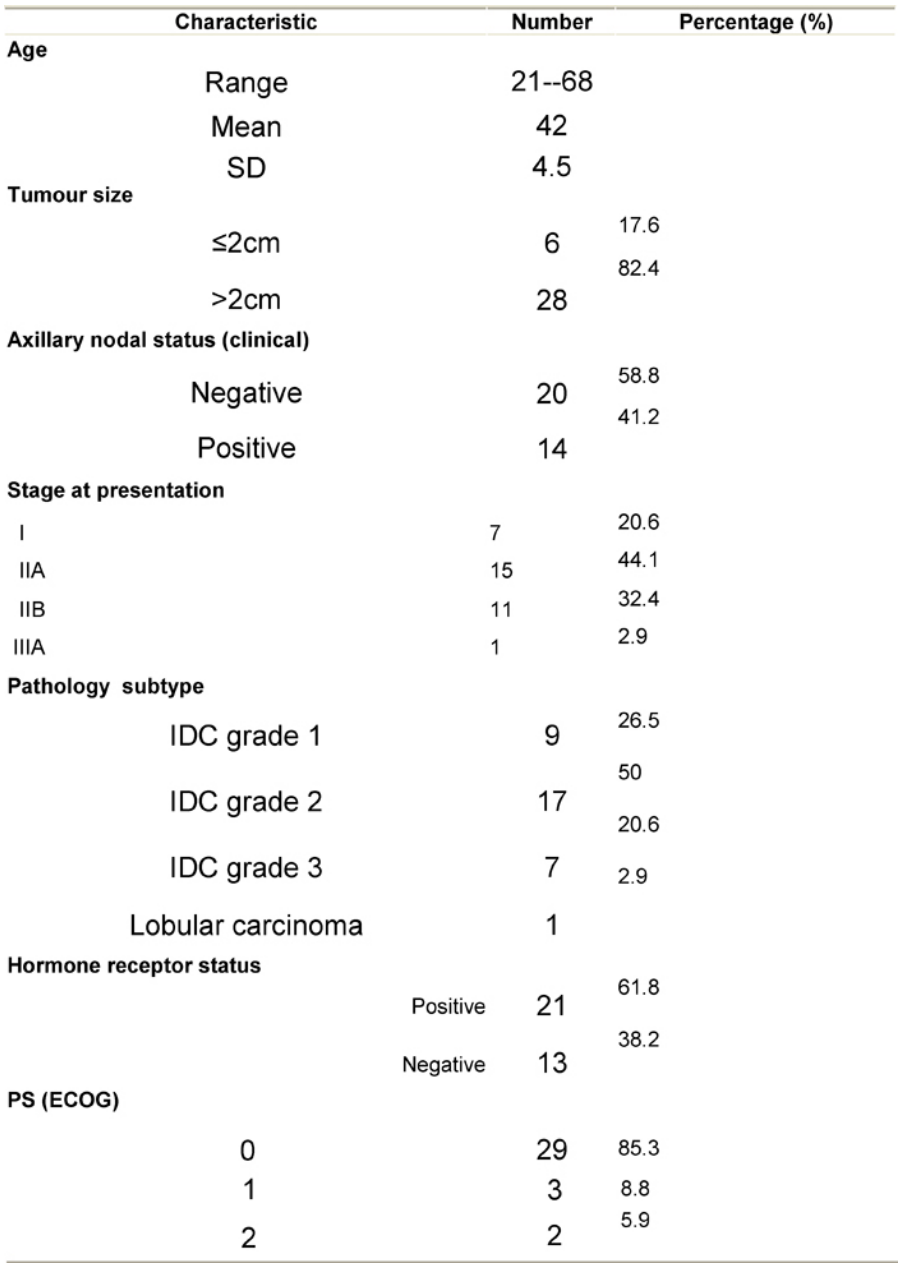

saline ( $\mathrm{pH}$ 6.0) and boiled for 20 minutes. Endogenous peroxidase activity was blocked by incubation with $6 \% \mathrm{H}_{2} \mathrm{O}_{2}$ in methanol. The primary antibodies used were mouse monoclonal anti-human oestrogen receptor alpha, clone 1D5 (ready to use), mouse monoclonal anti-human progesterone receptor, clone PgR 636 (ready to use) and concentrated rabbit polyclonal antihuman C-erb-B-2 oncoprotein (Her2/neu) (1:50), Hercept test, Code No. A0485 (Dako, Copenhagen, Denmark). The primary antibodies were incubated overnight. Immunoreactivity was visualized using Envision + (Dako cytomation, Glostrup, Denmark) with DAB chromogen as substrate and Mayer's haematoxylin as counterstain. Previous positive breast carcinoma for ER, PR and C-erb-B-2 were used as positive control for ER, PR and C-erb-B-2, respectively. Negative controls were prepared by substituting the primary antibodies with saline.
Evaluation of immunostaining: nuclear staining was a prerequisite for assigning ER and PR positivity; both the number of tumour cell nuclei and the intensity of the reaction were evaluated. For C-erb-B-2, complete membranous staining in more than $10 \%$ was required for positivity.

\section{Results}

Thirty-four patients were enrolled in this study, patient characteristics are shown in Table 1.

All patients received the planned chemotherapy in time except for two patients who had severe neutopenia requiring colony stimulating factors and treatment delay (one patient delayed for one week and the other for ten days). 
Table 2: Chemotherapy toxicity profile for the 34 patients of the study

\begin{tabular}{|c|c|c|}
\hline Toxicity & Number & Percentage \\
\hline \multicolumn{3}{|l|}{ Cardiac function } \\
\hline \multicolumn{3}{|l|}{ No dysfunction } \\
\hline \multirow{2}{*}{$\begin{array}{l}\text { Asymptomatic } \\
\text { dysfunction }\end{array}$} & 30 & 88.3 \\
\hline & 3 & 8.8 \\
\hline $\begin{array}{l}\text { Symptomatic } \\
\text { dysfunction requiring } \\
\text { TTT }\end{array}$ & 1 & 2.9 \\
\hline \multicolumn{3}{|l|}{ Haematologic anemia } \\
\hline None/mild & 25 & 73.5 \\
\hline Moderate & 7 & 20.6 \\
\hline Severe & 2 & 5.9 \\
\hline \multicolumn{3}{|l|}{ Neutropenia } \\
\hline None/mild & 26 & 76.5 \\
\hline Moderate & 6 & 17.6 \\
\hline Severe & 2 & 5.9 \\
\hline \multicolumn{3}{|l|}{ GIT toxicities } \\
\hline \multicolumn{3}{|l|}{ Stomatitis } \\
\hline None & & 17.6 \\
\hline Transient & 6 & 67.7 \\
\hline Severe & 23 & 14.7 \\
\hline \multicolumn{3}{|l|}{ Nausea } \\
\hline Transient & & 73.5 \\
\hline Severe & 25 & 26.5 \\
\hline \multicolumn{3}{|l|}{ Vomiting: } \\
\hline Transient & & 79.4 \\
\hline Severe & 27 & 20.6 \\
\hline \multicolumn{3}{|l|}{ Diarrhoea } \\
\hline None & & 82.3 \\
\hline$<2$ days & 28 & 11.8 \\
\hline \multirow[t]{2}{*}{$>2$ days } & 4 & 5.9 \\
\hline & 2 & \\
\hline \multicolumn{3}{|l|}{ Alopecia } \\
\hline None & 2 & 5.9 \\
\hline Moderate patchy & 3 & 8.8 \\
\hline Complete & 29 & 85.3 \\
\hline
\end{tabular}

Chemotherapy was well tolerated with acceptable toxicities.

Mild to moderate toxicity that is temporary and improves on simple and ordinary treatment is assigned as transient, while severe toxicity requiring specific aggressive treatment or admission is assigned as severe. Toxicity resulting from chemotherapy is shown in Table 2.

Tumour size of more than $2 \mathrm{~cm}$ was observed in 28 patients $(82.4 \%)$ at time of presentation, while only six patients (17.6\%) 
Table 3: Surgery

\begin{tabular}{|c|c|c|}
\hline Parameter & Number & Percentage \\
\hline \multicolumn{3}{|l|}{ Procedure } \\
\hline MRM & 11 & 32.4 \\
\hline $\mathrm{BCS}$ & 23 & 67.6 \\
\hline \multicolumn{3}{|l|}{ Tumour site } \\
\hline Upper outer & 18 & 53 \\
\hline Lower outer & 6 & 17.6 \\
\hline Central & 5 & 14.7 \\
\hline Inner & 5 & 14.7 \\
\hline \multicolumn{2}{|l|}{ BCs } & 100 \\
\hline Lumpectomy + axillary & 16 & 69.6 \\
\hline \multicolumn{3}{|l|}{ Dissection } \\
\hline Quadrantectomy + axillary dissection & 7 & 30.4 \\
\hline \multicolumn{2}{|l|}{ Complications } & 100 \\
\hline \multicolumn{3}{|l|}{ MRM } \\
\hline Seroma (axillary) & 3 & 8.8 \\
\hline Wound infection & 1 & 2.9 \\
\hline Total & $6 / 34$ & 17.6 \\
\hline \multicolumn{3}{|l|}{ Patient satisfaction with cosmesis } \\
\hline \multicolumn{3}{|l|}{ MRM N/A(11) } \\
\hline \multicolumn{3}{|l|}{ Lumpectomy(16) } \\
\hline Very good & 12 & 75 \\
\hline Good & 3 & 18.7 \\
\hline Fair & 1 & 6.3 \\
\hline \multicolumn{3}{|l|}{ Quadrantectomy (7) } \\
\hline Very good & 3 & 43 \\
\hline Good & 2 & 28.5 \\
\hline Fair & 2 & 28.5 \\
\hline \multicolumn{3}{|l|}{ Total (23) } \\
\hline Very good & 15 & 65 \\
\hline Good & 5 & 22 \\
\hline Fair & 3 & 13 \\
\hline
\end{tabular}

had a tumour size of $2 \mathrm{~cm}$ or less. After completion of the course of chemotherapy 23 patients (67.6\%) had tumours of 2 $\mathrm{cm}$ or less allowing less extensive resections.

\section{Surgery}

All patients underwent surgery within 35 days of the fourth cycle (average 19 days) in the form of conservative breast surgery in $23(67.6 \%)$ or modified radical mastectomy in 11 (32.4\%) patients.
Decision to perform breast conservative surgery (BCS) or modified radical mastectomy was based on the tumour size, breast tumour ratio and tumour position within the breast as shown in Table 3. Modified radical mastectomy was not followed by immediate reconstruction. Perioperative antibiotics were given to all patients and suction drains were used. Routine second day discharge from hospital with the drains was done for all patients. One lumpectomy patient was readmitted five days later for wider excision due to involved margins in the final report in spite of negative margins by frozen section. 
Table 4: Clinical tumour response after chemotherapy

\begin{tabular}{lcc}
\hline Clinical Response & Number & Percentage \\
Primary tumour & & \\
CR & 7 & 20.6 \\
PR & 17 & 50 \\
No change & 9 & 26.5 \\
\hline PD & 1 & 2.9 \\
Total & 34 & 100 \\
Lymph nodes & & \\
Negative & 28 & 82.4 \\
Positive & 6 & 17.6 \\
Total & 34 & 100
\end{tabular}

Of the seven patients who had a $C C R$, only four patients $(11.8 \%)$ had pCR. One patient was microscopically free of tumour at the primary site and axilla after chemotherapy but was not initially classified as having a CCR.

Table 5: Pathologic tumour response after chemotherapy

\begin{tabular}{lcc}
\hline Pathologic response & Number & Percentage \\
Primary tumour & & \\
pCR & 5 & 14.7 \\
No pCR & 29 & 85.3 \\
Total & 34 & 100 \\
\hline
\end{tabular}

In patients undergoing BCS wide excision, staining of frozen sections was performed to control the margins. A problem was met in patients with $\mathrm{CCR}$ where no palpable tumour was present. In these patients, a quadrantectomy was done where the index quadrant of the tumour was excised. All cases undergoing BCS underwent full axillary dissection through a separate transverse lower axillary crease incision.

Patients with a partial response lumpectomy was usually possible guided by the palpable lump although in three cases the breast mass was non-palpable and excision was performed after preoperative wire localization. Perioperative antibiotics were used for all patients, routine axillary suction drains were used while lumpectomy cavity drains were only used selectively. No mortalities were observed and only minor morbidity in the form of wound infection (two cases cleared spontaneously on treatment) and seroma (two cases in the mastectomy group required several aspirations and two cases of axillary seroma in the BCS group required aspiration and drain reinsertion in one case). The cosmetic results were evaluated as regard the appearance of the scars, the position and size of the areolanipple complex and the size of the breast in comparison with the other breast as very good $(+++)$, moderate $(++)$ or fair $(+)$.
In the BCS group, patient satisfaction with final cosmetic appearance was very satisfactory. The quadrantectomy patients were less satisfied with the final outcome than those undergoing lumpectomy.

\section{Tumour response}

An overall objective response was observed in $70.6 \%$ of the patients. Seven patients (20.6\%) experienced a cCR, 17 patients $(50.0 \%)$ a partial response, nine patients $(26.5 \%)$ had stable disease and one patient had disease progression (see Table 4).

Pathologic complete response $(\mathrm{pCR}=$ grade 5$)$ was seen in five (14.7\%) patients and a pPR (grade 1-4) in 29 patients (Table 5). There was no axillary lymph node involvement after chemotherapy in 26 patients (76.5\%), of them true negative (category A) represented (58.8\%) and signs of tumour regression (nodal fibrosis, mucin pools, aggregates of foamy histiocytes, myxoid or mucinous areas) were seen in five patients (category D). No response (category B) was observed in five patients (14.7\%) and nodes with metastasis associated 
Table 6: Pathologic tumour response (graded) after chemotherapy

\begin{tabular}{lccc}
\hline Site & $\begin{array}{l}\text { Grade of Pathological } \\
\text { Response }\end{array}$ & Number & Percentage \\
Primary tumour & pPR 1 & 3 & 8.8 \\
& 2 & 8 & 23.5 \\
& 3 & 15 & 44.1 \\
Total & 4 & 3 & 8.8 \\
& pCR 5 & 5 & 14.7 \\
Lymph node & & 34 & 100 \\
& A (true negativity) & 20 & 58.8 \\
& B (No response) & 5 & 14.7 \\
& C (pPR) & 3 & 8.8 \\
& D (pCR) & 6 & 17.6 \\
& & 34 & 100 \\
\hline
\end{tabular}

pPR: pathologic partial response; $\mathrm{PCR}$ : pathologic complete response.

with histological alterations were observed in three patients (8.8\%)

A tumour size of more than $2 \mathrm{~cm}$ was observed in 28 patients $(82.4 \%)$ at the time of presentation, while a tumour size of $2 \mathrm{~cm}$ or less was seen in only five patients (17.6\%). After completion of the course of chemotherapy, 23 patients (67.6\%) were observed to have tumours of $2 \mathrm{~cm}$ or less so allowing for less extensive resections. Pathologic examination of axillary nodes revealed negative nodes in 26 patients and positive nodes in eight patients. Patients with positive nodes showed signs of regression while signs of complete tumour necrosis (burnt-out tumour) were seen in five patients with negative nodes.

\section{Further management}

Patients who had a good biological (pathological) response received two more cycles of the same regimen (FEC100) to a total of six cycles. Those who had a poor biological response were shifted to a taxane-containing regimen (docitaxel and cisplatin). All patients who underwent BCS received radiotherapy to the residual breast and chest wall as well as peripheral lymphatics (when indicated). Doses of radiotherapy to the residual breast and chest wall were 5000cGy in 25 fractions over five weeks followed by a booster dose of 1500 to 2000 cGy in 7-10 fractions over 10-15 days. Hormonal therapy was given to hormone responsive tumours. Follow-up of patients ranged from 6 to 28 months. No local recurrences were seen in any of our patients during this limited follow-up. One case developed distant metastasis (lung) 14 months after completion of therapy.

\section{Discussion}

Neoadjuvant and adjuvant chemotherapy for breast cancer have been compared in several previous clinical trials, but these trials did not definitively show that one approach was better than the other. The rationale for the use of neoadjuvant chemotherapy is: (1) the ability to reduce the extent of surgical procedures so, allowing for better cosmoses, (2) the ability to assess clinical as well as biological response of the given treatment, (3) eradication of micro-metastases, (4) to allow precise pathologic assessment of tumour response and (5) an opportunity for a scientific study of serial pre- and posttreatment tumour biopsy samples [14].

The use of preoperative or primary chemotherapy was introduced approximately three decades ago in locally advanced breast cancer. Since then, its role in the management of locally advanced breast cancer has been firmly established. However, the advantages are not clear in early breast cancer 
[15]. Despite the fact that preoperative chemotherapy may permit more breast-conserving treatment modalities, there may be problems, for instance in achieving adequate loco-regional control as a result of the difficulty of assessing tumour margins after the administration of preoperative chemotherapy [16].

Objective clinical response to neoadjuvant chemotherapy is reported to be between $65 \%$ and $91 \%$. In our series, clinical response was seen in $70.6 \%$ of our cases, which is in accordance with other published reports $[17,18]$. Reported CCR rates are much lower ranging from $10 \%$ to $30 \%$ in most series, although some authors have reported a much lower rate. In our patients, CCR was seen in $20.6 \%$ of the cases, which is in accordance with most published reports [14]. Pathological complete responses are usually in the $9-15 \%$ range although there are some reports citing up to $30 \%$ pathological response rates. The reason for this discrepancy is usually because these authors include minimal disease (or only microscopic foci of disease) in their figures for CPR [19-21]. We were able to detect a cPR in $14.7 \%$ of our cases, which is also in agreement with most of the published data.

Axillary downstaging is also reported to be a good prognostic factor. A primary tumour response is usually associated with a good axillary response. In our patients, $41.2 \%$ had palpable axillary nodes on presentation. After the end of chemotherapy, only $26.5 \%$ had palpable nodes.

Although the benefits of preoperative chemotherapy in early breast cancer patients are less clear compared with the locally advanced breast cancer patients, the potential to enhance breast-conserving therapy makes it an attractive treatment modality. Several authors have firmly established the reduced need for mastectomy with neoadjuvant chemotherapy or neoadjuvant endocrine therapy $[19,22,23]$. In our experience, mastectomy was performed in only $32.4 \%$ of cases compared to a mastectomy rate of almost $80 \%$ observed at the Menofia University Cancer Center. This is in accordance with the finding of other authors who showed a significant reduction in the need for mastectomy; Schwartz et al [19] observed a $62 \%$ rate of breast conservation while Makaris et al reported an $89 \%$ rate of breast conservation with neoadjuvant chemotherapy compared to $78 \%$ when neoadjuvant chemotherapy was not used $[19,23]$.

Complications after surgery were only minimal with no significant differences between patients who underwent a mastectomy and those who had BCS. The two major problems encountered by us were: (1) the difficulty in assessing margins by frozen section in patients who had been given neoadjuvant chemotherapy; and (2) the accurate localization of the tumour site in patients with $\mathrm{CCR}$ or $\mathrm{CPR}$ with minimal disease. Kuerer et al recommend radiological placement of metallic markers prechemotherapy especially in women with tumours of less than 2 $\mathrm{cm}$ to facilitate adequate resection and pathologic processing [24]. Kurbet et al also advocate intra-operative evaluation of the margins [25]. We agree with this and believe it will permit easier and more accurate surgery. Cosmetic results and patient satisfaction in our experience were acceptable, although those who had a lumpectomy had better results than those who underwent a quadrantectomy.

Scholl et al and Mauriac et al both demonstrated in randomized trials a survival benefit for the neoadjuvant chemotherapy group $[26,27]$. Other studies, including the NSABP-18 and EORTC 10902 trial, were unable to show any significant difference in overall survival, disease-free survival or loco-regional control. This is supported by most other authors, none of whom were able to demonstrate a survival benefit; however all the trials were able to demonstrate objective clinical response and decrease in the rate of mastectomy. Moreover, this was not associated with an increase in local recurrence. In the EORTC 10902 trial, investigators compared preoperative versus postoperative chemotherapy in breast cancer, where they found that the breast-conserving therapy rate was higher in the preoperative chemotherapy group in comparison with the postoperative chemotherapy group. This finding, together with the equal loco-regional control rate in both groups, advocates the advantageous role of primary chemotherapy in breastconserving management $[9,14,23]$.

In large trials, although there was no overall survival difference between neoadjuvant and adjuvant chemotherapy arms, a difference in overall survival, disease-free survival and locoregional control was observed in several subsets of patients. Survival was more significantly improved in the subset of patients who achieved PCR than in the other subsets of patient and than those receiving adjuvant chemotherapy. Likewise, survival was significantly better in patients who had an axillary pCR than in those who did not. Kuerer et al went further and stratified patients into three groups. They found the worst survival was for patients with gross axillary disease postchemotherapy, followed by those with occult micro-metastases, and the best survival was recorded for patients with $\mathrm{pCR}$. These findings are supported by several other authors [28-30]. In our patients, the issues of survival and long-term loco-regional control were not addressed due to the low number of cases and the short follow-up period.

The chemotherapy regimen used in our study was well tolerated and not associated with any major side effects necessitating discontinuation of the treatment. This is in accordance with most 
other studies that reported the chemotherapy regimens were well tolerated with minimum morbidity $[14,18]$.

In locally advanced and primary inoperable breast cancer, the purpose of preoperative treatment is to enable adequate local treatment. In patients with stage I or II breast cancer who are candidates for breast-conserving therapy irrespective of preoperative chemotherapy, the goal of preoperative chemotherapy is unclear. However, in stage I or II breast cancer patients who are not candidates for BCS, preoperative chemotherapy will definitely increase the proportion who may avoid a mastectomy. Some investigators argue that tumour response to preoperative chemotherapy is an independent predictor of treatment outcome. Therefore, it could be of benefit for breast cancer patients to adjust systemic adjuvant treatment at an early stage if tumour response to preoperative chemotherapy is inadequate. Controversially, preoperative chemotherapy might lead to over-treatment of breast cancer patients. This can be explained by the fact that patients receive systemic treatment regardless of histological staging of the tumour and axillary nodal status.

Moreover, the possibility of studying the effects of chemotherapy on well-established tumour characteristics as well as experimental tumour markers makes chemotherapy in the preoperative setting highly attractive for research purposes [31]. The comparison of core needle biopsies with the same tumour after systemic treatment is a worthwhile reason to continue preoperative chemotherapy trials in early breast cancer.
Unfortunately, not much data concerning quality-of-life issues in relation to preoperative chemotherapy are available in the literature. Quality-of-life studies, however, have been performed to investigate the effects of breast-conserving therapy versus mastectomy and show a less impaired body image for the conservative treatment modality [32]. Considering the fact that preoperative as well as post-operative chemotherapy seem to yield similar results in terms of prognosis, this might be a conclusive factor on the decision of which chemotherapeutic strategy should be chosen. Therefore, the role of preoperative chemotherapy should be studied in future trials that focus on research, equivalence, quality of life, and local control, in addition to better prognosis in patient subsets.

\section{Conclusion}

The use of neoadjuvant chemotherapy in operable breast cancer in this study was associated with tumour and axillary downstaging, which increased the proportion of cases undergoing breast conservation, with acceptable side effects and reasonable cost. The results from our study are in accordance with the most important studies looking at the same question. During the limited follow-up time of this study, no locoregional recurrences were recorded and one distant treatment failure was recorded. Its impact, if any, on overall or diseasefree survival was not addressed in this study. Larger multicentre randomized studies with a long follow-up are needed to compare the overall and disease-free survival benefit of this treatment modality, especially in different subtypes. 


\section{References}

1. (1998) Polychemotherapy for early breast cancer: An overview of the randomized trials. Early Breast Cancer Trialists' Collaborative Group Lancet 352 930-42 PMID 9752815 10.1016/S0140-6736(98)03301-7

2. Goldhirsch A, Glick JH, Gelber RD et al (1998) Meeting highlights: International consensus panel on the treatment of primary breast cancer J Natl Cancer Inst 90 1601-8 PMID 9811309 10.1093/inci/90.21.1601

3. Hortobagyi GN, Ames FC, Buzdar AU et al (1988) Management of stage III primary breast cancer with primary chemotherapy, surgery, and radiation therapy Cancer 62 2507-16 PMID 3056604 10.1002/1097-0142 (19881215)62:123.0.CO;2-D

4. Bonadonna G, Veronesi U, Brambilla C et al (1990) Primary chemotherapy to avoid mastectomy in tumours with diameters of three centimeters or more $\mathrm{J}$ Natl Cancer Inst 82 1539-45 PMID 2402015 10.1093/ inci/82.19.1539

5. Fisher B, Gunduz N and Saffer E (1983) Influence of the interval between primary tumour removal and chemotherapy on kinetics and growth metastases Cancer Res 43 1488-92 PMID 6831397

6. Fisher B, Gunduz N, Coyle J et al (1989) Presence of a growth-stimulating factor in serum following primary tumour removal in mice Cancer Res 49 1996-2001 PMID 2702641

7. Fisher B, Saffer E, Rudock C et al (1989) Effect of local or systemic treatment prior to primary tumour removal on the production and response to a serum growthstimulating factor in mice Cancer Res 49 2002-4 PMID $\underline{2522814}$

8. Goldie JH and Coldman AJ (1979) A mathematic model for relating the drug sensitivity of tumours to their spontaneous mutation rate Cancer Treat Rep 63 172733 PMID 526911

9. Fisher B, Brown A, Mamounas E et al (1997) Effect of preoperative therapy for primary breast cancer (BQ) on local-regional disease, disease-free survival (DFS) and survival (S): Results from NSABP B-18 Proc Annu Meet Am Soc Clin Oncol 16127449 (abstract)

10. EORTC Breast Cancer Cooperative Group (1998) Manual for Clinical Research in Breast Cancer (Almere, The Netherlands: Excerpta Medica) pp 96-97

11. Miller AB, Hoogstraten B, Staquet $M$ and Winkler A (1981) Reporting results of cancer treatment Cancer 47 207-14 PMID 7459811 10.1002/1097-0142(19810101)47:13.0.CO; $\underline{2-6}$
12. (2005) Pathology Reporting of Breast Disease NHSBSP Publication no. 58 (Sheffield: NHS Publications)

13. Smith IC, Heys SD, Hutcheon AW et al (2002) Neoadjuvant chemotherapy in breast cancer: significantly enhanced response with docetaxel $\mathrm{J}$ Clin Oncol 20 1456-66 PMID 11896092 10.1200/JCO.20.6. $\underline{1456}$

14. van der Hage JA, van de Velde $\mathrm{CJH}$, Julien J, TubianaHulin M, Vandervelden C and Duchateau L (2001) Preoperative Chemotherapy in Primary Operable Breast Cancer: Results From the European Organization for Research and Treatment of Cancer Trial 10902 J Clin Oncol 1922 4224-37 PMID 11709566

15. Lerouge D, Touboul E, Lefranc JP, Genestie C, MoureauZabotto L and Blondon J (2004) Locally advanced non inflammatory breast cancer treated by combined chemotherapy and preoperative irradiation: updated results in a series of 120 patients Cancer Radiother 83 155-67 PMID 15217583

16. Pierga JY, Mouret E, Laurence V et al (2003) Prognostic factors for survival after neoadjuvant chemotherapy in operable breastcancer. The role of clinical response Eur J Cancer 39 1089-96 PMID 12736108 10.1016/S09598049(03)00069-8

17. Mauriac L, MacGrogan G, Avril A et al (1999) Neoadjuvant chemotherapy for operable breast carcinoma larger than $3 \mathrm{~cm}$ : A unicentre randomized trial with a 124month median follow-up Ann Oncol 10 47-52 PMID 10076721 10.1023/A:1008337009350

18. Fisher B, Bryant J, Wolmark $\mathrm{N}$ et al (1998) Effect of preoperative chemotherapy on the outcome of women with operable breast cancer J Clin Oncol 16 2672-85 PMID 9704717

19. Schwartz GF, Meltzer AJ, Lucarelli EA, Cantor JP and Curcillo PG 2nd (2005) Breast conservation after neoadjuvant chemotherapy for stage II carcinoma of the breast J Am Coll Surg 2013 327-34 PMID 16125064 10.1016/j.jamcollsurg.2005.03.015

20. Powles TJ, Hickish TF, Makris A et al (1995) Randomized trial of chemoendocrine therapy started before or after surgery for treatment of primary breast cancer $\mathrm{J}$ Clin Oncol 13 547-52 PMID 7884414

21. Van Praagh I, Cure H, Leduc B, Charrier S, Le Bouedec G, Achard JL et al (2002) Efficacy of a primary chemotherapy regimen combining vinorelbine, epirubicin, and methotrexate (VEM) as neoadjuvant treatment in 89 patients with operable breast cancer Oncologist $7 \quad 5 \quad 418-23$ PMID 12401904 10.1634/ theoncologist.7-5-418 
22. Clahsen PC, van de Velde CJH, Julien JP et al (1996) Improved local control and disease-free survival after perioperative chemotherapy for early-stage breast cancer J Clin Oncol 14 745-53 PMID 8622020

23. Makris A, Powles TJ, Ashley SE et al (1998) A reduction in the requirements for mastectomy in a randomized trial of neoadjuvant chemoendocrine therapy in breast cancer Ann Oncol 9 1179-84 PMID 9862047 10.1023/A: 1008400706949

24. Kuerer HM, Singletary SE, Buzdar AU, Ames FC, Valero V, Buchholz TA et al (2001) Surgical conservation planning after neoadjuvant chemotherapy for stage II and operable stage III breast carcinoma Am J Surg 1826 601-8 PMID 11839324 10.1016/S0002-9610(01)00793-0

25. Kurbet S, de Barros AC and Pinotti JA (2006) The safe association of intraoperative evaluation of surgical margins and neoadjuvant chemotherapy in breast cancer larger than 3 centimeters Tumouri $92121-5$ PMID 16683380

26. Scholl SM, Fourquet A, Asselain B et al (1994) Neoadjuvant versus adjuvant chemotherapy in premenopausal patients with tumours considered too large for breast conserving surgery: Preliminary results of a randomized trial: S6 Eur J Cancer 30A 64552 PMID 8080680 10.1016/0959-8049(94)90537-1

27. Mauriac L, Durand M, Avril A et al (1991) Effects of primary chemotherapy in conservative treatment of breast cancer patients with operable tumours larger than $3 \mathrm{~cm}$ Ann Oncol 2 347-54 PMID 1954179
28. Kuerer HM, Sahin AA, Hunt KK, Newman LA, Breslin TM, Ames FC et al (1999) Incidence and impact of documented eradication of breast cancer axillary lymph node metastases before surgery in patients treated with neoadjuvant chemotherapy Ann Surg 2301 72-8 PMID 10400039

29. Cure H, Amat S, Penault-Llorca F et al (2002) Prognostic value of residual node involvement in operable breast cancer after induction chemotherapy Breast Cancer Res Treat 76 37-45 PMID 12408374 10.1023/A: 1020274709327

30. Newman LA, Pernick NL, Adsay V et al (2003) Histopathologic evidence of tumour regression in the axillary lymph nodes of patients treated with preoperative chemotherapy correlates with breast cancer outcome Ann Surg Oncol 10 734-9 PMID $\underline{12900363}$ 10.1245/ASO.2003.03.081

31. Clahsen PC, van de Velde CJH, Duval C et al (1999) The utility of mitotic index, estrogen receptor and Ki-67 measurements in the creation of novel prognostic indices for node-negative breast cancer Eur J Surg Cancer 25 356-63 PMID 10419704 10.1053/ejso.1999. $\underline{0657}$

32. Poulsen B, Graversen HP, Beckmann J et al (1997) A comparative study of post-operative psychosocial function in women with primary operable breast cancer randomized to breast conservation therapy or mastectomy Eur J Surg Cancer 23 327-34 PMID 9315062 10.1016/S0748-7983(97)90804-0 